\title{
Detailed Characterization of Undeveloped Upwards Annular Flow using the Brightness Based Laser Induced Fluorescence Technique
}

\author{
João Vasques ${ }^{1}$, Mikhail Cherdantsev ${ }^{2}$, Andrey Cherdantsev ${ }^{2}$, David Hann ${ }^{1}$, Buddhika Hewakandamby ${ }^{1}$, \\ Barry Azzopardi', \\ ${ }^{1}$ Faculty of Engineering, University of Nottingham \\ University Park NG7 2RD, Nottingham, United Kingdom \\ joao.vasques@nottingham.ac.uk; cherdantsev@itp.nsc.ru \\ ${ }^{2}$ Kutateladze Institute of Thermophysics \\ 1 Lavrentiev Ave. 630090, Novosibirsk, Russia \\ cherdantsev@gmail.com
}

\section{Extended Abstract}

The interfacial wave structure of the liquid phase in upwards annular gas-liquid flow in a $11.7 \mathrm{~mm}$ pipe was studied using the brightness based laser induced fluorescent technique (BBLIF). Due to the versatility of the BBLIF technique, film thickness measurements were carried out over different axial locations covering a measurement area with very high spatial and temporal resolution. These comprise measurements performed close to the inlet section (between 0 to $10 \mathrm{z} / \mathrm{D}$ ), where the dynamics of the small wavelets that are created due to the gas-liquid interaction and that subsequently will lead to the formation of the disturbance waves was analysed. Furthermore, using a tracking algorithm to detect disturbance waves, a full characterization in terms of their velocity, distance and time separation (both in absolute and in terms of their distribution), frequency, intermittency and wavelength was carried out based on measurements conducted further downstream (between 28 to $37 \mathrm{z} / \mathrm{D}$ ). An Omega-K analysis was also conducted to these latter measurements showing a constant increasing ratio between the ripple and disturbance wave velocity.

Although these measurements were conducted well below the predicted length for a fully developed annular flow to occur, it was observed that the behaviour of the structures present in the film during these undeveloped stages plays a crucial role on the overall events that occur further downstream. 